\title{
MAXIMAL SUBGROUPS AND THE JORDAN-HÖLDER THEOREM
}

\author{
JULIO LAFUENTE
}

(Received 12 June 1987; revised 12 February 1988)

Communicated by $\mathrm{H}$. Lausch

\begin{abstract}
In this note we present a general Jordan-Hölder type theorem for modular lattices and apply it to obtain various (old and new) versions of the Jordan-Hölder Theorem for finite groups.

1980 Mathematics subject classification (Amer. Math. Soc.): 20 D 30.
\end{abstract}

Isbell [10] has observed that the Jordan-Hölder Theorem may be derived from the Zassenhaus Theorem, and that this yields a uniqueness statement for the correspondence given by the Jordan-Hölder Theorem. This result, however, does not give the various versions of the Jordan-Hölder Theorem for finite groups that have received some interest more recently, for example, the one that states that for any two chief series of a finite group a correspondence can be found associating Frattini chief factors with Frattini chief factors and non-Frattini ones with non-Frattini ones. Such a theorem was first published by Carter, Fischer and Hawkes [4] for finite soluble groups, and for finite groups in general in the author's [12], with a different approach by Förster in [7] (see also Chapter 1 of [2]). Further, Barnes proved that in soluble groups corresponding complemented (which, for finite soluble groups, means nonFrattini) chief factors have a common (maximal) complement. On the other hand, for arbitrary finite groups the number of complemented chief factors in a given chief series can depend on the series (see Baer and Förster [2] or Kovács and Newman [11] for examples).

(C) 1989 Australian Mathematical Society $0263-6115 / 89 \$ A 2.00+0.00$ 
Here we will obtain a Jordan-Hölder correspondence for chief series of an arbitrary finite group $G$ which not only respects the Frattini or non-Frattini nature of chief factors, but also the property of being complemented by a maximal subgroup; in fact, corresponding chief factors have a common maximal complement, if complemented at all by a maximal subgroup. (However, for such a correspondence, corresponding chief factors are not normally $G$ isomorphic, but only $G$-connected as defined by the author in [13] and, independently, by Förster in [7] ( $G$-related) and [2] ( $G$-verwandt).)

Our result will emerge as a corollary to a Jordan-Hölder type theorem for modular lattices, in an approach inspired by unpublished notes [11] of Kovács and Newman.

\section{A general Jordan-Hölder Theorem in modular lattices}

Throughout this section, $\mathscr{L}$ will denote a modular lattice, $\mathscr{M}$ a subset of the set $\mathscr{P}$ of its prime intervals (that is, those pairs $A, B$ of elements of $\mathscr{L}$ such that $B<A$, and $C \in\{A, B\}$ whenever $B \leq C \leq A$; we shall adopt the notation $A / B$ for such pairs), and $K=Y_{0}<Y_{1}<\cdots<Y_{n}=H$ will denote a chain in $\mathscr{L}$ such that $Y_{i} / Y_{i-1} \in \mathscr{P}, i=1, \ldots, n$. We set $\mathscr{L}_{K, H}=\{X \in \mathscr{L} \mid K \leq X \leq H\}$ and $\mathscr{P}_{K, H}=\{X / Y \in \mathscr{P} \mid K \leq Y$ and $X \leq H\}$.

Further, we write $A / B \gg X / Y$ (or $X / Y \ll A / B$ ), if $A / B, X / Y \in \mathscr{P}$ are such that $A=X \vee B$ and $X \wedge B=Y$. If $X^{*} / X \ll Z^{*} / Z \gg Y^{*} / Y$ or $X^{*} / X \gg Z^{*} / Z \ll Y^{*} / Y$ for some $Z^{*} / Z$, we say that $X^{*} / X$ and $Y^{*} / Y$ are under the Zassenhaus correspondence: $X^{*} / X$ Zsh $Y^{*} / Y$. (General notation and terminology will be taken from [9].)

The following observation (and its dual, which we omit) is well known.

1.1 Lemma. For any $X^{*} / X \in \mathscr{P}_{K, H}$ there exists some $j \in\{1, \ldots, n\}$ such that $X^{*} \vee Y_{k}=X \vee Y_{k}$ for $k=j, \ldots, n, X^{*} \vee Y_{k}>X \vee Y_{k}$ for $k=0, \ldots, j-1$ and

$$
X^{*} \vee Y_{j-1} / X \vee Y_{j-1} \gg X^{*} \vee Y_{j-2} / X \vee Y_{j-2} \gg \cdots \gg X^{*} \vee Y_{0} / X \vee Y_{0}=X^{*} / X
$$

1.2 Definitions. (a) Two prime intervals $R_{i} / S_{i}, i=1,2$, are said to be of the same $\mathscr{M}$-type, if either both are in $\mathscr{M}$ or both are in $\mathscr{M}^{\prime}=\mathscr{P} \backslash \mathscr{M}$.

(b) If $\mathscr{M} \ni C / D \ll A / B \in \mathscr{M}^{\prime}$ and $A / C \in \mathscr{P}$, then $(A / B, C / D)$ is an M-crossing.

(c) $\mathscr{A}$ is called an $M$-set in $\mathscr{L}$, if it satisfies the following two conditions.

(M1) If $A / B \gg C / D$, then $A / B \in \mathscr{M}$ implies that $C / D \in \mathscr{M}$.

(M2) If $(A / B, C / D)$ is an $\mathscr{C}$-crossing, then so is $(A / C, B / D)$. 
Note that $\mathscr{M}$ is an $M$-set in $\mathscr{L}$ if and only if $\mathscr{K}^{\prime}$ is an $M$-set in the dual of $\mathscr{L}$. Trivial examples of $M$-sets are given by $\mathscr{P}$ and $\varnothing$. We record a simple property of $M$-sets, leaving the verification (as well as the statement of the dual) to the reader.

1.3 Lemma. Let $X^{*} / X \in \mathscr{M} \subseteq \mathscr{P}_{K, H}$ and set $Y^{*}=Y_{j}, Y=Y_{j-1}$ where $j=\max \left\{i \in\{1, \ldots, n\} \mid X^{*} \vee Y_{i-1} / X \vee Y_{i-1} \in \mathscr{M}\right\}$. Then one of the following holds.

(i) $X^{*} \vee Y^{*}=X \vee Y^{*}=X^{*} \vee Y, X^{*} / X \ll X^{*} \vee Y^{*} / X \vee Y \gg Y^{*} / Y$, $X \wedge Y=X^{*} \wedge Y=X \wedge Y^{*}$ and $X^{*} / X \gg X^{*} \wedge Y^{*} / X \wedge Y \ll Y^{*} / Y$.

(ii) $\left(X^{*} \vee Y^{*} / X \vee Y^{*}, X^{*} \vee Y / X \vee Y\right)$ is an $\mathscr{M}$-crossing, $X^{*} / X \ll$ $X^{*} \vee Y / X \vee Y$ and $Y^{*} / Y \ll X \vee Y^{*} / X \vee Y$.

In particular, if $\mathscr{M}$ is an $M$-set, then in both cases $Y^{*} / Y \in \mathscr{M}$, and the same holds for $X^{*} \vee Y^{*} / X \vee Y$ and $X \vee Y^{*} / X \vee Y$.

In the remainder of this section, $\mathscr{M}$ will always denote an $M$-set in $\mathscr{L}$.

1.4 Definition. Two prime intervals $X^{*} / X$ and $Y^{*} / Y$ are $\mathscr{M}$-related, if one of the following holds.

(1) $X^{*} / X \ll R^{*} / R \gg Y^{*} / Y$ for some $R^{*} / R \in \mathscr{M}$.

(2) $X^{*} / X \ll B / D$ and $C / D \gg Y^{*} / Y$ for some $\mathscr{K}$-crossing $(A / B, C / D)$.

(3) $X^{*} / X \gg S^{*} / S \ll Y^{*} / Y$ for some $S^{*} / S \in \mathscr{M}^{\prime}$.

(4) $X^{*} / X \gg A / B$ and $A / C \ll Y^{*} / Y$ for some $\mathscr{M}$-crossing $(A / B, C / D)$.

1.5 TheOREM. Let $\mathscr{L}$ be a modular lattice and $\mathscr{M}$ an $M$-set in $\mathscr{L}_{K, H}$. Assume that

$$
K=X_{0}<X_{1}<\cdots<X_{n}=H \text { and } K=Y_{0}<Y_{1}<\cdots<Y_{m}=H
$$

are two maximal chains in $\mathscr{L}$ between $H$ and $K$. Then $n=m$, and there exists a unique $\pi \in S_{n}$ such that $X_{i} / X_{i-1}$ and $Y_{i^{\pi}} / Y_{i^{\pi}-1}$ are $\mathscr{M}$-related for $i=1, \ldots, n$.

In fact,

$$
\begin{gathered}
i^{\pi}=\max \left\{j \in\{1, \ldots, n\} \mid X_{i} \vee Y_{j-1} / X_{i-1} \vee Y_{j-1} \in \mathscr{M}\right\}, \quad \text { if } X_{i} / X_{i-1} \in \mathscr{M}, \\
i^{\pi}=\min \left\{j \in\{1, \ldots, n\} \mid X_{i} \wedge Y_{j} / X_{i-1} \wedge Y_{j} \in \mathscr{M}^{\prime}\right\}, \quad \text { if } X_{i} / X_{i-1} \in \mathscr{M}^{\prime} .
\end{gathered}
$$

PROof. Without loss of generality, $m \leq n$. Let the map $\pi:\{1, \ldots, n\} \rightarrow$ $\{1, \ldots, m\}$ be defined by the equations in the statement of the theorem.

First note that applying 1.3 and its dual to the definition of $\pi$ one sees that $X_{i} / X_{i-1}$ and $Y_{i^{\pi}} / Y_{i^{\pi}-1}$ are $\mathscr{K}$-related for $i=1, \ldots, n$ and, therefore, have the same $\mathscr{M}$-type. 
In order to prove injectivity, and hence bijectivity, of $\pi$, write $X^{*} / X=$ $X_{i} / X_{i-1}, Y^{*} / Y=Y_{j} / Y_{j-1}$, and $Z^{*} / Z=X_{k} / X_{k-1}$, where $i<k$, but $i^{\pi}=$ $j=k^{\pi}$. Suppose that $X_{i} / X_{i-1} \in \mathscr{M}$; thus, all three intervals are $\mathscr{M}$-intervals. Now apply Lemma 1.3 .

In the first case, $X^{*} \vee Y^{*}=X^{*} \vee Y$. From $X^{*} \leq Z$ we get that $Z \vee Y^{*}=Z \vee Y$. Since $Z^{*} / Z \in \mathscr{M}$ and $k^{\pi}=j$, Lemma 1.3 applies and yields the contradiction that $Z \vee Y^{*}>Z \vee Y$.

Hence $\left(X^{*} \vee Y^{*} / X \vee Y^{*}, X^{*} \vee Y / X \vee Y\right)$ is an $\mathscr{M}$-crossing; so $X^{*} \vee Y^{*} / X^{*} \vee$ $Y \in \mathscr{M}^{\prime}$. Since $Z \vee Y \neq Z^{*} \vee Y$ by Lemma $1.3, Z \vee Y^{*} / Z \vee Y \in \mathscr{P}$. As $X^{*} \leq Z$ gives $X^{*} \vee Y \leq Z \vee Y$ as well as $X^{*} \vee Y^{*} \leq Z \vee Y^{*}$, we may use (M1) to deduce that $Z \vee Y^{*} / Z \vee Y \in \mathscr{M}^{\prime}$, contrary to the conclusion of Lemma 1.3.

We have shown that the restriction of $\pi$ to $I=\left\{i \in\{1, \ldots, n\} \mid X_{i} / X_{i-1} \in\right.$ $\mathscr{M}\}$ is injective. Application of this conclusion to the dual of $\mathscr{L}$, with $\mathscr{M}^{\prime}$ instead of $\mathscr{M}$, shows that the restriction of $\pi$ to $\{1, \ldots, n\} \backslash I$ is injective. As mentioned above, $\pi$ leaves these two sets invariant, and we may conclude that $\pi$ is injective.

Finally, if $\psi$ is any permutation with the above properties, then the definition of $\pi$ requires that $i^{\psi} \leq i^{\pi}\left(i^{\psi} \geq i^{\pi}\right)$ for all $i \in I(i \in\{1, \ldots, n\} \backslash I)$. Consequently, $\psi=\pi$.

Taking $\mathscr{M}=\mathscr{P}$ gives Isbell's result, with the Zassenhaus correspondence: here Condition 1.4(1) always applies, and there are no $\mathscr{M}$-crossings. Somewhat more general, under the following hypothesis $(*)$, conditions $(2)$ and (4) in Definition 1.4 are redundant:

$(A / B, C / D)$ is an $\mathscr{M}$-crossing in $\mathscr{L}$ implies $A / E \in \mathscr{M}$ for some $E \in \mathscr{L}$ with $D<E<A$.

Observe that, for $A, B, C, D, E$ as in (*), by Theorem 1.5, applied to $\mathscr{L}_{D, A}, A / E \in \mathscr{M}$ implies that $E / D \in \mathscr{M}^{\prime}$; furthermore, if $X^{*} / X \ll B / D$, $C / D \gg Y^{*} / Y$, then we have $X^{*} / X \ll A / E \gg Y^{*} / Y$ (and, of course, the dual statement also holds).

It is easy to see that, under the hypothesis of Theorem 1.5 , to a given $X_{i} / X_{i-1}$ there may exist more than one $Y_{k} / Y_{k-1} \mathscr{M}$-related to $X_{i} / X_{i-1}$. However, one always has

1.6 Proposition. Assume the hypothesis of Theorem 1.5 and let $\Pi$ be any theoretical property on $\mathscr{P}_{K, H}$ which is preserved under the relation of being A -related.

Then for any $X_{i} / X_{i-1}$ with $\Pi$, there exists at least one $X_{j} / X_{j-1}$ with $\Pi$ and of the same $\mathscr{M}$-type as $X_{i} / X_{i-1}$, which is $\mathscr{M}$-related to only one $Y_{k} / Y_{k-1}$.

Proof. We consider the case $X_{i} / X_{i-1} \in \mathscr{M}$. Let us define $k=\min \left\{k^{\prime} \in\{1, \ldots, n\} \mid Y_{k^{\prime}} / Y_{k^{\prime}-1}\right.$ has $\Pi$ and is in $\left.\mathscr{M}\right\}$, 
and write $k=j^{\pi}$ with $\pi$ given by Theorem 1.5. From Theorem 1.5, $X_{j} / X_{j-1}$ is $\mathscr{M}$-related to $Y_{k} / Y_{k-1}$; in particular, it has $\Pi$ and belongs to $\mathscr{M}$.

Assume that $X_{j} / X_{j-1}$ is $\mathscr{M}$-related to $Y_{k^{\prime}} / Y_{k^{\prime}-1}$. Then the latter, like $X_{j} / X_{j-1}$, has $\Pi$ and is in $\mathscr{M}$; so $k \leq k^{\prime}$ by choice of $k$. On the other hand, $k=j^{\pi}$ is maximal with respect to $X_{j} \vee Y_{k-1} / X_{j-1} \vee Y_{k-1} \in \mathscr{M}$, so that $k \geq k^{\prime}$ is a consequence of the following general observation (which is easily derived from Definition 1.4, using (M1)): if $X_{j} / X_{j-1}$ is $\mathscr{M}$-related to $Y_{k^{\prime}} / Y_{k^{\prime}-1}$, and is in $\mathscr{M}$, then $X_{j} \vee Y_{k^{\prime}-1} / X_{j-1} \vee Y_{k^{\prime}-1} \in \mathscr{M}$.

\section{Applications to finite groups}

In this section we consider chief series of a finite group $G$ (and $K, H \unlhd G$, $K \leq H)$.

(1) Since the lattice $\mathscr{L}$ of normal subgroups of a group is modular, we may apply Theorem 1.5 to deduce Isbell's version of the Jordan-Hölder Theorem for finite groups (namely, by taking $\mathscr{M}=\mathscr{P}_{K, H}$ ). This yields a correspondence $\pi$ between the chief factors of the two series such that for all $i$ the corresponding factors $X_{i} / X_{i-1}$ and $Y_{i^{\pi}} / Y_{i^{\pi}-1}$ satisfy $X_{i} / X_{i-1}$ Zsh $Y_{i^{\pi}} / Y_{i^{\pi}-1}$ and, in particular, are $G$-isomorphic.

(2) To get the Carter, Fischer and Hawkes version mentioned in the introduction (but for not necessarily soluble finite groups), with a correspondence $\pi_{\Phi}$ respecting the Frattini or non-Frattini nature of corresponding chief factors, one considers the set $\mathscr{M}_{\Phi}$ of all non-Frattini chief factors between $K$ and $H$ (the chief factors supplemented in $G$ by a proper subgroup of $G$ ). This is an $M$-set: indeed, condition (M1) is trivial, while (M2) follows from two basic properties of the Frattini subgroup (see, for example, 1.25 in [2], for the less well-known one of them); in fact, the latter property also proves the validity of hypothesis (*) from Section 1 ; so $\mathscr{M}_{\Phi}$-related chief factors $A / B$ and $C / D$ always satisfy $A / B \mathrm{Zsh} C / D$, and hence are $G$-isomorphic.

(3) Let $\mathscr{S}$ be any set of maximal subgroups of $G$ and consider the set $\mathscr{M}_{S}$ of all those chief factors $X / Y$ of $G$ complemented in $G$ by at least one element $U$ of $\mathscr{S}$ :

$$
G=U X \text { and } U \cap X=Y .
$$

Again $\mathscr{K}_{S}$ satisfies (M1), but (M2) does not hold generally; for example, if $G$ is elementary abelian of order $r,\{A, B, C\}$ the set of its maximal subgroups and $\mathscr{S}=\{A, B\}$, then all chief factors of $G$ except $G / C$ are complemented by some $U$ in $\mathscr{S}$; thus $(G / C, B / 1)$ is an $\mathscr{M}_{S}$-crossing, but $(G / B, C / 1)$ is not.

A similar example, but with the relevant chief factors being non-abelian, is given by $G=E_{1} \times E_{2} \times E_{3}$ where $E_{1}, E_{2}, E_{3}$ are any three isomorphic non-abelian simple groups, with $\mathscr{S}=\left\{D_{12} \times E_{3}, D_{23} \times E_{1}\right\}$ where $D_{i j}$ is a 
diagonal subgroup of $E_{i} \times E_{j}$. Here $\left(E_{2} \times E_{3} / E_{2}, E_{3} / 1\right)$ is an $\mathscr{M}_{S}$-crossing, but $\left(E_{2} \times E_{3} / E_{3}, E_{2} / 1\right)$ is not.

Yet another type of counterexample is obtained as follows. Let $G \in \mathscr{P}_{\Pi}^{\prime}$, the class of all groups $G$ with a maximal subgroup $U$ such that $\operatorname{Core}_{G}(U)$, the normal core of $U$ in $G$, is 1 and, $\mathbf{S}(G)$, the socle in $G$, is a non-abelian minimal normal subgroup of $G$ complemented by $U$. (For examples of such groups see Förster [6]; a description of all groups in $\mathscr{P}_{\Pi}^{\prime}$ can be found in Förster [8].) Let $S \cong_{G} \mathrm{~S}(G)$ and form the semidirect product $H=G S$. This has precisely two minimal normal subgroups: $S$, and a diagonal subgroup $T$ of $\mathbf{S}(G) \times S$, and these are complemented by $G$ (see, for example, the first sections in Baer [1], Förster [5]). Now let $\mathscr{S}=\{G, U T\}$. Then all chief factors of $G$ below $T \times S$ except $(T \times S) / S$ are complemented.

These three examples suggest the hypothesis (\#) on $\mathscr{S}$ stated below. This hypothesis is not necessary for $\mathscr{K}_{\mathscr{S}}$ to satisfy (M2) in the lattice $\mathscr{L}_{1, G}$ (it is satisfied, though, by those $\mathscr{M}_{\mathscr{S}}$ we are interested in), but it appears to be difficult to formulate in a satisfactory manner the precise condition on $\mathscr{S}$ for $\mathscr{M}_{\mathscr{S}}$ to satisfy (M2). Before stating (\#), we recall from Baer and Förster [2], Förster [7], Lafuente [13], the definition of the crown $C / R$ of a group $G$ associated with its non-Frattini chief factor $X / Y$ :

$C=X \mathbf{C}_{G}(X / Y), R=\bigcap_{U \in \mathscr{T}} \operatorname{Core}_{G}(U), \mathscr{T}$ the set of all maximal subgroups $U$ of $G$ such that $X / Y$ is $G$-isomorphic to a minimal normal subgroup of $G / \operatorname{Core}_{G}(U)$.

(\#) For each crown $C / R$ of $G$ and any two chief factors $X_{i} / Y_{i}$ of $G$ such that $R \leq Y_{i}$ and $X_{i} \leq C(i=1,2)$, if $X_{1} / Y_{1}$ has a complement in $G$ from $\mathscr{S}$, then so does $X_{2} / Y_{2}$, except, perhaps, when $X_{i}=C \neq X_{3-i}$ for some $i \in\{1,2\}$.

(We do not require that the chief factors have a common complement.)

Basic properties of crowns are described in $[2,7,13]$, and will be used without further reference. From such properties the following is immediate.

(+) Let $X / Y$ be a chief factor of $G$ and $C / R$ the crown of $G$ associated with it, and let $U$ be a maximal subgroup of $G$. Then $U$ complements $X / Y$ if and only if $U$ complements $X R / Y R$.

Using (+), we will deduce a Jordan-Hölder Theorem for general $\mathscr{M}_{\mathscr{S}}$ from the special case where the lattice $\mathscr{L}_{K, H}$ involved is $\mathscr{L}_{R, C}$. So we now assume that $\mathscr{S}$ consists of maximal subgroups $U$ of $G$ complementing a chief factor of $G$ between $R$ and $C$; in view of the structure of crowns (cf. 2.4 in [7]), this means that $U$ complements a minimal normal subgroup of $G /$ Core $_{G}(U)$ and $R \leq \operatorname{Core}_{G}(U) \leq C$. 
Assume hypothesis (\#). In order to verify condition (M2) for $\mathscr{M}_{\mathscr{S}}$, let

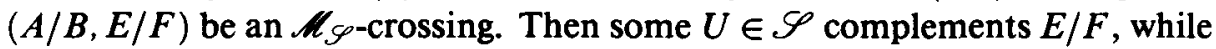
$A / B$ does not have a complement in $\mathscr{S}$; in particular, $U$ cannot complement $A / B$. Now $U \cap B>F$ would easily lead to the contradiction that $U \cap A=$ $U \cap B=B$. Thus $U \cap B=F$ and $B \not U$; so $U$ complements $B / F$. (In fact, we could have infered from (\#) the existence of a complement of $U$ from $\mathscr{S}$.) It remains to observe that from (\#) it follows that $A / E$ is not complemented by an element of $\mathscr{S}$ : since $A / B$ is not complemented by an element of $\mathscr{S}$, but $B / F<C / F$ is, (\#) requires that $A=C$; and then, if $A / E$ were complemented by an element of $\mathscr{S}$, the same should apply to $A / B$.

Next, let $\mathscr{S}_{C / R}$ be the set of all maximal subgroups complementing a chief factor between $R$ and $C$. Recall that all chief factors $X / Y$ of $G$ between $R$ and $C$ are isomorphic (although all of them are $G$-isomorphic only if $C / R$ is abelian or is itself a chief factor of $G$; however, they are always similar in the sense of 53.11 of [15], and $G$-connected/ $G$-related in the sense of [13] and [7]. Observe that all these chief factors $X / Y$ are complemented in $G$ by a maximal subgroup, except those for which $X=C$ and $G / Y \notin \mathscr{P}_{\Pi}^{\prime}$. Actually, in [14] we have pointed out that each non-soluble finite group $G$ has a crown $C / R$ such that the [pairwise isomorphic] groups $G / T, T \unlhd G$ with $R \leq T \leq C$ and $C / T$ a chief factor, are not in $\left.\mathscr{P}_{\Pi}^{\prime}\right)$. Evidently, the set $\mathscr{S}_{C / R}$ satisfies hypothesis (\#) irrespective of whether or not the crown is complemented (that is, the $G / T, T \unlhd G$ with $R \leq T \leq C$ and $C / T$ a chief factor, are in $\mathscr{P}_{n}^{\prime}$ or not). Hence the above discussion together with Theorem 1.5 yields a Jordan-Hölder correspondence $\pi_{C / R}$, and a uniqueness statement for this.

(4) To get the general result, note that each chief factor $X / Y$ of $G$ is either Frattini or has a unique crown $C / R$ associated with it. The latter is determined by the requirement that $X R<Y R$ (and then $X / Y \ll X R / Y R$; in fact, $X / Y \cong_{G} X R / Y R$ ). Therefore, given any chief series of $G$, multiplying by $R$ induces a bijection between those factors in the series whose associated crown is $C / R$ and the factors in the chief series of $G$ between $R$ and $C$ obtained by taking the images of the former chief factors under such multiplication. Now put $\mathscr{C}_{\Phi}=\mathscr{M}_{\Phi}$, the set of all Frattini chief factors of $G$ and, for each crown $C / R$ of $G$, let $\mathscr{E}_{C / R}$ comprise all nonFrattini chief factors of $G$ with $C / R$ as their associated crown. Define $\mathscr{M}_{C / R}=\mathscr{M}_{\mathscr{S}}$ where $\mathscr{S}=\mathscr{S}_{C / R}$, and note that $\mathscr{M}_{C / R} \subseteq \mathscr{E}_{C / R}$. Also, say that two chief factors are $\mathscr{M}$-related, if both belong to the same $\mathscr{C}_{x}$ and are $\mathscr{M}_{x}$-related, where $x$ is $\Phi$ or some $C / R$. Finally, given two chief series of $G$ of lengths $n, m$, define $\pi \in S_{n}$ by requiring that the restriction of $\pi$ to $I_{x}=\left\{i \in\{1, \ldots, n\} \mid H_{i} / K_{i} \in \mathscr{C}_{x}\right\}$, where $x=\Phi$ or $x=C / R$ for some crown $C / R$ of $G$, be $\pi_{x}$. Then from (2) and (3) we obtain (most of) our main result 
(which we formulate for $\mathscr{L}_{K, H}$, although our proof here dealt only with the special case $K=1$ and $H=G$ ).

2.1 Theorem. Let $K=X_{0}<X_{1}<\cdots<X_{n}=H$ and $K=Y_{0}<Y_{1}<$ $\cdots<Y_{m}=H$ be two chief series of $G$ between $H$ and $K$. Then $n=m$, and there exists a unique $\pi \in S_{n}$ such that $X_{i} / X_{i-1}$ and $Y_{i^{\pi}} / Y_{i^{\pi}-1}$ are M-related for $i=1, \ldots, n$. This means the following.

(i) $Y_{i^{\pi}} / Y_{i^{\pi}-1} \leq \Phi\left(G / Y_{i^{\pi}-1}\right) \Leftrightarrow X_{i} / X_{i-1} \leq \Phi\left(G / X_{i-1}\right) \Leftrightarrow X_{i} / X_{i-1} \cong_{G}$ $Y_{i^{n}} / Y_{i^{\pi}-1}$; in fact, there is a Frattini factor $A / B$ such that $X_{i} / X_{i-1} \gg A / B \ll$ $Y_{i^{\pi}} / Y_{i^{\pi}-1}$.

(ii) $X_{i} / X_{i-1} \not \Phi\left(G / X_{i-1}\right) \Leftrightarrow X_{i} / X_{i-1}$ is $G$-connected to $Y_{i^{n}} / Y_{i^{n}-1}$.

(iii) $Y_{i^{\pi}} / Y_{i^{\star}-1}$ is complemented in $G$ by a maximal subgroup $\Leftrightarrow X_{i} / X_{i-1}$ is complemented in $G$ by a maximal subgroup $\Rightarrow X_{i} / X_{i-1}$ and $Y_{i^{\pi}} / Y_{i^{\pi}-1}$ have a common maximal complement in $G$, and for the crown $C / R$ of $G$ associated with $X_{i} / X_{i-1}$, either $X_{i} / X_{i-1} \ll A / B \gg Y_{i^{\pi}} / Y_{i^{\pi}-1}$ for some chief factor $A / B$ of $G$ between $R$ and $C$ (in particular, $X_{i} / X_{i-1} \cong_{G} Y_{i^{\pi}} / Y_{i^{\pi}-1}$ ), or $X_{i} / X_{i-1} \ll C / T_{i} \neq C / S_{i} \gg Y_{i^{\pi}} / Y_{i^{\star}-1}$ where $T_{i}, S_{i} \unlhd G$ contain $R$ and are such that $C / T_{i}$ and $C / S_{i}$ are non-complemented chief factors of $G$.

(iv) $X_{i} / X_{i-1}$ is non-Frattini, but not complemented by a maximal subgroup $\Rightarrow X_{i} R=C=Y_{i \pi} R$ and $C / X_{i-1} R$ and $C / Y_{i^{\pi}-1} R$ are non-complemented chief factors of $G$, where $C / R$ is the crown of $G$ associated with $X_{i} / X_{i-1}$. Moreover, for each $x \in\{\Phi\} \cup\{C / R \mid C / R$ a crown of $G\}$ and all $i \in\{1, \ldots, n\}$,

$i^{\pi}=\max \left\{j \in\{1, \ldots, n\} \mid X_{i} Y_{j-1} / X_{i-1} Y_{j-1} \in \mathscr{M}_{x}\right\}, \quad$ if $X_{i} / X_{i-1} \in \mathscr{M}_{x}$,

$i^{\pi}=\min \left\{j \in\{1, \ldots, n\} \mid X_{i} \cap Y_{j} / X_{i-1} \cap Y_{j} \in \mathscr{C}_{x} \backslash \mathscr{M}_{x}\right\}, \quad$ if $X_{i} / X_{i-1} \in \mathscr{C}_{x} \backslash \mathscr{M}_{x}$.

To check the above conditions (iii) and (iv), apply Definition 1.4 (here only cases $(1,2)$ can be relevant) together with statement $(+)$ above and the description of the structure of $G / R$ for a crown $C / R$ given in $[7,24]$.

\section{Acknowledgement}

I am grateful to P. Förster, L. G. Kovács and M. F. Newman for the opportunity to see $[2,7,8,11]$, and to the referee for the helpful comments.

\section{References}

[1] R. Baer, 'Classes of finite groups and their properties', Illinois J. Math. 1 (1987), 115-187.

[2] R. Baer and P. Förster, Einbettungsrelationen und Formationen endlicher Gruppen, to appear.

[3] D. W. Barnes, 'On complemented chief factors of finite soluble groups', Bull. Austral. Math. Soc. 7 (1972), 101-104. 
[4] R. W. Carter, B. Fischer and T. O. Hawkes, 'Extreme classses of finite soluble groups', $J$. Algebra 9 (1968), 285-313.

[5] P. Förster, 'Projektive Klassen endlicher Gruppen. l', Math. Z. 186 (1984), 149-178.

[6] P. Förster, 'A note on primitive groups with small maximal subgroups', Publ. Sec. Mat. Univ. Autonoma Barcelona 28 (1984), 19-28.

[7] P. Förster, 'Chief factors, crowns, and the generalized Jordan-Hölder Theorem', Comm. Algebra, to appear.

[8] P. Förster, 'On primitive groups with regular normal subgroups', ANU-MSRC Research Report 39-1985, Canberra.

[9] G. Grätzer, General lattice theory (Birkhäuser-Verlag, Basel, 1978).

[10] J. R. Isbell, 'Zassenhaus' Theorem supersedes the Jordan-Hölder Theorem', Adv. in Math. 31 (1979), 101-103.

[11] L. G. Kovács and M. F. Newman, 'On the Jordan-Hölder Theorem', ANU-MSRC Research Report 11, 1987, Canberra.

[12] J. Lafuente, 'Homomorphs and formations of given derived class', Math. Proc. Cambridge Philos. Soc. 84 (1978), 437-441.

[13] J. Lafuente, 'Nonabelian crowns and Schunck classes of finite groups', Arch. Math. 42 (1984), 32-39.

[14] J. Lafuente, 'Grupos primitivos con subgrupos maximales pequeños', Publ. Sec. Mat. Univ. Autonoma Barcelona 29 (1985), 154-161.

[15] H. Neumann, Varieties of groups (Springer-Verlag, Berlin, Heidelberg, New York, 1967).

Departamento de Matemáticas

Universidad de Zaragoza

50009 Zaragoza

Spain 